\title{
A study of pregnancy rates in "cleared" male factor couples
}

\author{
Erica S. Godart ${ }^{1}$, Daniel H. Shin ${ }^{2}$, Emily Christensen ${ }^{1}$, Eli R. Thompson ${ }^{3}$, Paul J. Turek ${ }^{1}$ \\ ${ }^{1}$ The Turek Clinic, Beverly Hills, CA, USA; ${ }^{2}$ Southern California Permanente Medical Group, Los Angeles, CA, USA; ${ }^{3}$ Northwest Urology LLC, \\ Portland, OR, USA \\ Contributions: (I) Conception and design: ES Godart, DH Shin, PJ Turek; (II) Administrative support: ES Godart, E Christensen, ER Thompson, \\ PJ Turek; (III) Provision of study materials or patients: ES Godart, E Christensen, PJ Turek; (IV) Collection and assembly of data: DH Shin, ER \\ Thompson, E Christensen, ES Godart; (V) Data analysis and interpretation: All authors; (VI) Manuscript writing: All authors; (VII) Final approval of \\ manuscript: All authors. \\ Correspondence to: Paul J. Turek, MD. 9033 Wilshire Blvd, Suite 408, Beverly Hills, CA 90211, USA. Email: DrPaulTurek@gmail.com.
}

Background: Among couples with male factor infertility, the natural pregnancy rates associated with classic male factor treatments are well described. In couples with unexplained infertility, the proportion due to occult male factor is unclear. We hypothesized that many men diagnosed with unexplained infertility are actually fertile. We describe the 1-year natural pregnancy rates among couples in whom the male partner has been "cleared" of infertility on urologic evaluation.

Methods: Consecutive infertile couples were recruited from a single practice (PJT) over a 3-year period. A thorough male factor evaluation was performed, including a history, physical examination and 2 semen analyses. Based on this assessment, male partners in whom bulk semen parameters were normal were "cleared" from further evaluation. Lifestyle modifications were allowed, but no medical or surgical treatments were offered. The presence or absence of a female factor evaluation was not required for study inclusion. Subjects were followed for 12 months or until a pregnancy was achieved. Subjects were contacted via telesurvery 1-year later and pregnancy status ascertained. Simple descriptive statistics were used to evaluate the significance of observations.

Results: Fifty-four men were enrolled in the study. The mean duration of infertility was 1.5 years (range, 0.4 to 4.0 years) and the mean male and female partner ages were 38.6 and 35.1 years, respectively. On evaluation, $40 \%$ of men were noted to have significant fertility risks that included a clinical varicocele, exposures, and androgen altering medications. Among n=31 couples with known pregnancy outcomes, 20/31 (65\%) conceived naturally at a mean of 9 months after evaluation (range, 3-30 mos). Another 1/31 (3\%) couples conceived with intrauterine insemination (IUI) and 4/31 (13\%) conceived with IVF-ICSI.

Conclusions: A significant proportion of men diagnosed with unexplained infertility have lifestyle risk factors on urologic evaluation. Care in the form of counseling at-risk patients regarding lifestyle issues, in the absence of formal treatment, may have value in improving the fertility potential in this population. Indeed, natural conception rates among men identified with unexplained infertility are substantial and suggest that many of these men are truly fertile.

Keywords: Idiopathic infertility; unexplained infertility; male infertility; semen analysis; pregnancy rate

Submitted Sep 07, 2020. Accepted for publication Dec 03, 2020.

doi: $10.21037 /$ tau-20-1240

View this article at: http://dx.doi.org/10.21037/tau-20-1240

\section{Introduction}

Approximately $85 \%$ of couples who attempt to conceive will succeed within one year (1). Among those who fail, more than half will have an identifiable male factor (2).
This population is substantial, with $9.4 \%$ of U.S. men aged 25-44 years reporting use of infertility services (3).

Upon evaluation, semen analysis abnormalities will be revealed in almost $60 \%$ of infertile men, while the 
Table 1 Telesurvey questions used to assess reproductive outcomes
1. Since you saw $\mathrm{Dr}$. for an infertility evaluation, have you achieved a pregnancy?
2. If yes, what was the outcome from, or date of birth of, that pregnancy?
3. If yes, was that pregnancy achieved naturally or with the help of assisted reproduction (IUI, IVF)?
4. If no, have you tried assisted reproductive techniques (IUI, IVF, ICSI) in addition to at-home attempts to conceive?
5. If no, and if assisted reproduction was used, what technology (IUI vs. IVF vs. IVF-ICSI) and how many attempts of each were tried?

IVF, in vitro fertilization.

remainder will show normal semen quality (3). When a thorough male factor evaluation is performed in the setting of a normal semen analysis (4), it is also common that the history and physical exam are similarly unremarkable. Such infertility cases are designated as unexplained in nature and further evaluation with more sophisticated semen testing, including antisperm antibodies or sperm DNA fragmentation assays, are considered (5). Overall, it is estimated that a more subtle male factor underlies about $25-30 \%$ of unexplained infertility cases $(6,7)$. Typically, whether or not an etiology is revealed, affected couples will proceed to either intrauterine insemination (IUI) or in vitro fertilization (IVF) as the next therapeutic step.

However, another interpretation of an entirely normal male factor evaluation is that there is, in fact, no male fertility impairment. Indeed, if $25-30 \%$ of unexplained infertility is male-derived, then the vast majority of men categorized as having unexplained infertility may very well be "fertile". We hypothesize that a significant proportion of men deemed to have unexplained infertility are actually normally fertile. Since the natural history of fertility in this cohort of men is not well described, we studied the pregnancy rates in a cohort of men who were formally evaluated and "cleared" of male factor infertility. We present the following article in accordance with the TREND reporting checklist (available at: http://dx.doi.org/10.21037/tau-20-1240).

\section{Methods}

\section{Study subjects}

The study consists of consecutive infertile couples recruited from a single male infertility practice (PJT) over a 3-year period. The male factor infertility evaluation included a thorough history, a complete physical examination and at least 2 semen analyses (5). Semen analyses were performed according to World Health Organization reference ranges (8). Any and all prior general medical, hormonal metabolic laboratory studies were reviewed. Based on this formal assessment, male partners in whom the bulk semen parameters were normal on at least one of two semen samples by WHO standards and in whom no significant infertility risks factors were observed based on history and physical examination, were "cleared" from further medical evaluation. Lifestyle modifications were allowed following the male evaluation. As such, men who took antioxidant supplements and those who discontinued tobacco, finasteride, hot baths and saunas, qualified for study followup. However, those who received formal medical or surgical therapy for male factor issues were excluded from the study. The presence or absence of a female factor evaluation was not required for study inclusion. Subjects were followed for 12 months or until a pregnancy was achieved.

\section{Survey technique}

\section{Patient contact}

Identified subjects were contacted and consented through a telephone interview, according to HIPAA guidelines for patient contact. During the interview, the subject was provided with informed consent and HIPAA documentation and verbal consent obtained. Consent documents were then mailed to subjects for written signature with return envelopes included. During the tele-interview, the study goals were explained and a survey administered (Table 1). The total length of the phone interview was 5 minutes.

\section{Data de-identification}

After demographic and clinical information was collected from the electronic medical record and patient interviews, the data was formally de-identified for analysis. The study was conducted in accordance with the Declaration of Helsinki (as revised in 2013). The study was approved by Health Sciences Institutional Review Board at University of Southern California (HS-13-00578) and informed consent was taken from all the patients. 
Table 2 Demographic information of subjects and partners

\begin{tabular}{lcc}
\hline Demographic variable & Mean & Range \\
\hline Duration of infertility (years) & 1.5 & $0.4-4$ \\
Male age (years) & 38.6 & $31-49$ \\
Female age (years) & 35.1 & $25-44$ \\
\hline
\end{tabular}

Table 3 Fertility risk factors revealed by clinical history among study subjects $(\mathrm{n}=54)$

\begin{tabular}{lll}
\hline Risk factor & $\mathrm{n}$ & $\%$ \\
\hline Clinical varicocele (grades II or III) & 19 & $38 \%$ \\
Wet heat (Sauna, hot tub) & 15 & $28 \%$ \\
Tobacco use & 5 & $9 \%$ \\
Finasteride use & 2 & $4 \%$ \\
Excessive alcohol consumption (>2 drinks/day) & 2 & $4 \%$ \\
Proportion of subjects with infertility risk factors (includes overlap) & 22 & $40 \%$ \\
\hline
\end{tabular}

\section{Statistical analysis}

The primary outcome of the study was pregnancies achieved after male factor evaluation. Secondary outcomes include analyses of clinical and laboratory findings in study subjects. Simple descriptive statistics, including two tailed $t$-tests, were used to evaluate the significance of observations.

\section{Results}

\section{Demographic findings}

A total of 54 subjects were enrolled in the study over a 3 -year period. Demographic information about the subjects and their partners is outlined in Table 2. Upon clinical presentation, the mean duration of infertility was 1.5 years (range, 0.4 to 4.0 years) and the mean male and female partner ages were 38.6 and 35.1 years respectively. The vast majority of patients $(85 \%)$ presented with primary infertility and the remainder demonstrated secondary infertility.

\section{Clinical findings}

On the clinical history of study subjects, $40 \%$ were noted to have significant fertility risks that included a clinical varicocele or lifestyle exposures for infertility, including hot baths or saunas, tobacco or alcohol abuse, and the use of androgen altering medications such as finasteride (Table 3). At the time of the initial evaluation, they were advised to alter lifestyle issues to optimize fertility and encouraged to take antioxidant supplements. On physical examination, $38 \%$ presented with grade II or III left varicoceles; however, no other masses were present in any subject. No varicocele treatments were offered or performed on study subjects during the study follow-up period.

Semen parameters on routine analysis were within normal range for at least one of two semen analyses obtained from each study subject (Table 4). The first semen analysis was performed before the initial infertility evaluation, and the second sample was performed subsequent to this evaluation. The mean interval between two semen analyses performed on individuals was 8.8 weeks (range, 1-28 weeks). Interestingly, despite the fact that all semen analyses were considered technically "normal", there was a trend toward improved semen quality between the first and second semen samples (Table 4), driven mainly by an increase in sperm motility. This led to a significant improvement in total motile sperm counts between samples (65.8 million sperm to 150.4 million sperm, $\mathrm{P}<0.05$ ).

\section{Pregnancy outcomes}

As illustrated in Figure 1, among n=31 couples with known pregnancy outcomes, 20/31 (65\%) conceived naturally at a mean of 9 months after evaluation (range, 3-30 mos). Another 1/31 (3\%) couples conceived with IUI and 4/31 (13\%) conceived with IVF-ICSI. If reproductive assistance was used, 
Table 4 Comparison of paired semen analyses among study subjects

\begin{tabular}{lccc}
\hline Semen parameter & First, mean (range) & Second, mean (range) & $P$ \\
\hline Ejaculate volume $(\mathrm{mL})$ & $2.8(0.5-7)$ & $3.0(1-6.5)$ & 0.6 \\
Sperm density (millions $/ \mathrm{mL})$ & $68.4[5-286]$ & $80.0[19-366]$ & 0.4 \\
Total sperm count (millions) & $180.5(14.4-417.5)$ & $242.6(28.5-1,729)$ & 5.2 \\
Sperm motility (\%) & $49.2[11-72]$ & $57.4[21-77]$ & $<0.05$ \\
Total motile count (millions) & $65.8[8-237]$ & $150.4(17.6-1,193)$ & $<0.05$ \\
\hline
\end{tabular}

$\mathrm{mL}$, milliliters.

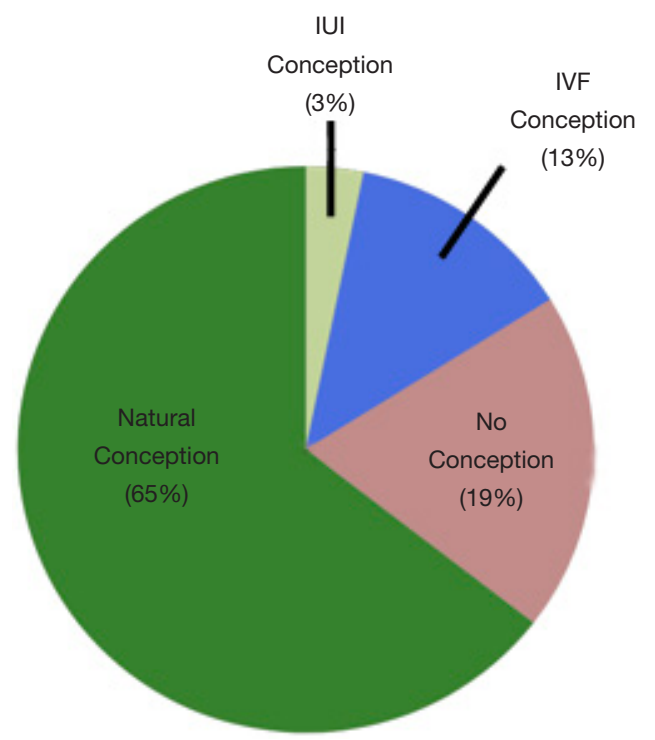

Figure 1 Mode of conception after a "cleared" male factor evaluation. Among n=31 patients who conceived (81\%) after a male infertility evaluation, the breakdown of how conceptions occurred are illustrated. IUI, intrauterine insemination; IVF, in vitro fertilization.

time to conception was longer at 22.3 months (range, 8-53). The remaining 6 couples had not conceived by one year.

\section{Conclusions}

We investigated the natural and assisted fertility rates among a small cohort of infertile couples who demonstrated no evidence of male factor infertility on a standard urologic evaluation. Indeed, this subset of men conceived naturally at a robust rate $(65 \%)$ after the male factor evaluation and did so without specific medical or surgical intervention. Another $16 \%$ of couples conceived with either IUI or IVF within a year of follow-up. Notably, this conception rate is markedly higher than the $10-15 \%$ pregnancy rate that is expected for untreated couples who are followed for a second year after an infertility diagnosis (9). These pregnancy rates compare favorably to other classic medical or surgical treatments offered to infertile men (Table 5). It also suggests that the male factor evaluation is reasonably robust in identifying relevant male factor issues that may impact fertility and that this straightforward evaluation may be a better predictor of true male fertility than previously believed. As such, the word "unexplained" may be inappropriate for many men with unremarkable male factor evaluations, as they may actually be fertile. Indeed, this pilot study suggests that twothirds of these men may be perfectly fertile.

There is another possible interpretation of these findings. Although men were "cleared" of male factor issues after a standard evaluation and were not offered formal medical or surgical treatment, they may have pursued lifestyle modifications that augmented natural fertility. Discontinuation of wet heat exposures (15), stopping medications such as finasteride, and reducing habitual alcohol or other drug use may have factored into improving the natural fertility of study subjects. In addition, dietary changes that increased antioxidant intake and the regular use of antioxidant supplements by study subjects could influence fertility potential (16). Although not quantified in this study, the effects of such lifestyle modifications are hard to quantify but likely contributed to improved fertility potential of men in the study. Given this alternative interpretation of the study findings, it is provocative to suggest that simple lifestyle modifications could substantially improve male fertility potential, but very reasonable to suggest that lifestyle issues merit further study for their potential to improve the fertility of men.

It is reassuring to see that the prevalence of varicoceles detected in this study cohort approximates what one would expect for an unselected population of men with infertility (17). More novel is the finding that $40 \%$ of infertile men had 
Table 5 Male fertility treatments and associated pregnancy rates

\begin{tabular}{lcc}
\hline Treatment & Pregnancy rates & Reference \\
\hline Varicocelectomy & $30-45 \%$ & $(10)$ \\
Vasectomy reversal & $30-60 \%$ & $(11)$ \\
TURED & $20-40 \%$ & $(12)$ \\
Clomiphene citrate & $15 \%$ & $(13)$ \\
IVF/ICSI & $4-50 \%$ & $(14)$ \\
\hline
\end{tabular}

IVF, in vitro fertilization.

what could be considered significant lifestyle risk factors, independent of varicocele, revealed on urologic evaluation. Although no malignancies were detected, the ability of the urologic assessment to detect clinical risk for male infertility is clearly apparent from the study. Indeed, the burden of lifestyle risk identified among infertile men supports the common medical idiom for other significant human diseases, like cancer or metabolic syndrome, that "lifestyle matters".

One statistically significant finding in the study was that the semen quality following an initial male factor evaluation improved compared to the intake semen analysis. Given that the mean time from the initial to subsequent semen analyses was almost 9 weeks, this could represent real clinical improvement following lifestyle modifications recommended at the initial visit. However, it may also reflect the great variability that exists within intraindividual semen analyses over time, and between different laboratories $(18,19)$. Therefore, caution is advised when evaluating the true underlying significance of the semen analysis findings.

Of course, significant study limitations exist, the foremost among them being limited patient numbers. Indeed, this may limit the ability to generalize the study findings. An accurate characterization of potential female factor issues was also not undertaken, although excluding couples based on defined female factors would likely have increased the proportion of those achieving pregnancies by making the denominator smaller. In addition, we were not able to confirm exactly which lifestyle changes were pursued by patients subsequent to their male factor evaluation. Lastly, this study did not address the issue of sperm "quality" as may be garnered from assays such as sperm DNA fragmentation (20). However, it is our belief that including these assays would likely have refined the relatively "blunt" instrument that is the standard semen analysis in predicting whom among study subjects would be fertile $v s$. infertile at study completion (21).

In summary, a significant proportion of men diagnosed with unexplained infertility have lifestyle risk factors ascertainable on a standard urologic evaluation. Medical care in the form of counseling at risk patients regarding lifestyle issues, in the absence of formal medical or surgical treatment, may have value in improving the fertility potential in this population. Indeed, the natural conception rates among men identified with unexplained infertility can be substantial and suggest that many of these men have no male factor infertility.

\section{Acknowledgments}

Funding: None.

\section{Footnote}

Reporting Checklist: The authors have completed the TREND reporting checklist. Available at http://dx.doi. org/10.21037/tau-20-1240

Data Sharing Statement: Available at http://dx.doi. org/10.21037/tau-20-1240

Peer Review File: Available at http://dx.doi.org/10.21037/ tau-20-1240

Conflicts of Interest: All authors have completed the ICMJE uniform disclosure form (available at http://dx.doi. org/10.21037/tau-20-1240). The authors have no other conflicts of interest to declare.

Ethical Statement: The authors are accountable for all aspects of the work in ensuring that questions related to the accuracy or integrity of any part of the work are appropriately investigated and resolved. The study was conducted in accordance with the Declaration of Helsinki (as revised in 2013). The study was approved by Health Sciences Institutional Review Board at University of Southern California (HS-13-00578) and informed consent was taken from all the patients.

Open Access Statement: This is an Open Access article distributed in accordance with the Creative Commons Attribution-NonCommercial-NoDerivs 4.0 International License (CC BY-NC-ND 4.0), which permits the noncommercial replication and distribution of the article with the strict proviso that no changes or edits are made and the original work is properly cited (including links to both 
the formal publication through the relevant DOI and the license). See: https://creativecommons.org/licenses/by-nc$\mathrm{nd} / 4.0 /$.

\section{References}

1. Infertility: A Tabulation of Available Data on Prevalence of Primary and Secondary Infertility. Geneva, Switzerland: WHO Programme on Maternal and Child Health and Family Planning, Division of Family Health. World Health Organization 1991.

2. Thonneau P, Marchand S, Tallec A, et al. Incidence and main causes of infertility in a resident population $(1,850,000)$ of three French regions (1988-1989). Hum Reprod 1991;6:811-16.

3. Chandra A, Copen CE, Stephen EH. Infertility service use in the United States: data from the National Survey of Family Growth, 1982-2010. Natl Health Stat Report 2014;(73):1-21.

4. Practice Committee of the American Society for Reproductive Medicine. Diagnostic evaluation of the infertile male: a committee opinion. Fertil Steril 2015;103: e18-25.

5. Turek PJ. Practical approach to the diagnosis and management of male infertility. Nat Clin Pract Urol 2005;2:226-38.

6. Mackenna A, Barratt CLR, Kessepoulu E, et al. The contribution of a hidden male factor to unexplained infertility. Fertil Steril 1993;59:405-11.

7. Hamada A, Esteves SC, Nizza M, et al. Unexplained male infertility: Diagnosis and management. Int Braz J Urol 2012;38:576-94.

8. Cooper TG, Noonan E, Eckardstein S, et al. World Health Organization reference values for human semen characteristics. Hum Reprod Update 2010;16:231-45.

9. Snick HK, Snick TS, Ever JL, et al. The spontaneous pregnancy prognosis in untreated subfertile couples: The Walcheren primary care study. Hum Reprod 1997;12:1582-8.

10. Çayan S, Shavakhabov S and Kadioğlu A. Treatment of

Cite this article as: Godart ES, Shin DH, Christensen E, Thompson ER, Turek PJ. A study of pregnancy rates in "cleared" male factor couples. Transl Androl Urol 2021;10(2):620625. doi: 10.21037/tau-20-1240 palpable varicocele in infertile men: A meta-analysis to define the best technique. J Androl 2009;30:33-40.

11. Belker AM, Thomas AJ, Fuchs EF, et al. Results of 1,469 microsurgical vasectomy reversals by the vasovasostomy study group. J Urol 1991;145:505-11.

12. Kadioglu A, Cayan S, Tefekli A, et al. Does response to treatment of ejaculatory duct obstruction in infertile men vary with pathology? Fertil Steril 2001;76:138-42.

13. Vandekerckhove P, Lilford R, Vail A, et al. WITHDRAWN: Clomiphene or tamoxifen for idiopathic oligo/asthenospermia. Cochrane Database Syst Rev 2007;(4):CD000151. PubMed

14. Sart.org. Survey of 2017 U.S. IVF pregnancy rates. Available online: https://www.sartcorsonline.com/rptCSR_ PublicMultYear.aspx? reportingYear=2017

15. Shefi S, Tarapore PE, Walsh TJ, et al. Wet heat exposure: a potentially reversible cause of low semen quality in infertile men. International Braz J Urol 2007;33:50-6; discussion 6-7.

16. Smits RM, Mackenzie-Proctor R, Yazdani A, et al. Antioxidants for male subfertility. The Cochrane database of systematic reviews. 13 March 2019. Available online: https://www.cochrane.org/CD007411/MENSTR_ antioxidants-male-subfertility PubMed

17. Gorelick JI, Goldstein M. Loss of fertility in men with varicocele. Fertil Steril 1993;59:613-6.

18. Auger J, Eustache F, Ducot B, et al. Intra-and interindividual variability in human sperm concentration, motility and vitality assessment during a workshop involving ten laboratories. Hum Reprod 2000;15:2360-8.

19. Leushuis E, van der Steeg JW, Steures P, et al. Reproducibility and reliability of repeated semen analyses in male partners of subfertile couples. Fertil Steril 2010;94:2631-5.

20. McReynolds S, Dzieciatkowska M, Stevens J, et al. Toward the identification of a subset of unexplained infertility: a sperm proteomic approach. Fertil Steril 2014;102:692-9.

21. Turek PJ. Does the male infertility clinical evaluation adequately assess toxicologic exposures? Fertil Steril 2008;89:suppl E69. 